\title{
Le beurre de karité au Burkina Faso : entre marché domestique et filières d'exportation
}

\author{
Martine François ${ }^{1}$ \\ Nathalie Niculescu ${ }^{1}$ \\ Zacharie Badini $^{2}$ \\ Mamadou Diarra ${ }^{3}$ \\ 1 Gret
}

45 bis, avenue de la belle Gabrielle 94736 Nogent sur Marne cedex <francois@gret.org>

2 SICAREX

01 BP 262513, rue 1

08 Ouagadougou

Burkina Faso

<sicarex@fasonet.bf>

3 DICSA

Korofina nord

rue 640 , porte 150

BP 2386

Bamako

Mali

<mmdiarra@icsahel.org>

\begin{abstract}
Résumé
Le beurre de karité fait partie des produits, à cheval entre usages domestiques et marchés d'exportation, pour lesquels une nouvelle demande internationale émerge. Il est issu d'une activité de cueillette et d'un long et pénible travail de transformation par les femmes rurales des zones soudano-sahéliennes. Il est aujourd'hui fortement valorisé en cosmétique, biotechnologies et agro-alimentaire. La demande internationale concerne essentiellement les noix de karité, mais aussi le beurre, pour lequel émerge une nouvelle demande de petites entreprises cosmétiques des pays développés, en rapport avec le commerce équitable. Les groupements de femmes sont les principaux acteurs de la transformation du karité en beurre. Ils répondent à cette demande internationale, qui reste limitée en volume à $10 \%$ du marché local. La concurrence entre groupements est féroce pour gagner les marchés internationaux. À quelles conditions une articulation "gagnant-gagnant" entre les acteurs africains des filières et ceux des pays développés est-elle possible? Les groupements de femmes semblent être en mesure de profiter de ces échanges. Les échanges internationaux permettent en effet aux groupements de femmes d'acquérir progressivement de meilleures capacités de négociation avec les acheteurs internationaux, en même temps que la capacité logistique permettant de fournir la qualité et les volumes requis dans les délais fixés par le client. Toutefois, il serait souhaitable qu'il existe une pression effective des consommateurs pour que le mot "équitable "soit précisément défini, qu'il permette effectivement une meilleure valorisation du travail à la base, et qu'existe la capacité à le vérifier. Ces opportunités qui portent sur de petites quantités et la concurrence exacerbée entre groupements ne doivent pas handicaper la structuration de la filière au niveau local. Ces conclusions appellent à un processus d'appui aux groupements de femmes pour une meilleure maîtrise de cette filière : transformation, négociation, produits, qualité - tant pour les marchés d'exportation que pour les marchés locaux et les marchés des pays voisins, plus accessibles et moins risqués.
\end{abstract}

Mots clés : beurre de karité ; Burkina Faso ; connaissance du marché ; exportation ; Karité (arbre).

Thèmes : économie et développement rural ; productions végétales ; technologie agroalimentaire ; transformation, commercialisation.

\section{Abstract \\ The shea butter market: between subsistence economy and globalised capitalism}

Shea butter is one of various products that fills a niche in both the domestic and export market and for which a new international demand is emerging. The product is the result of a collection activity and a long, labour-intensive processing operation conducted by rural women in the Sudan-Sahel zone. The product is now highly valued in the cosmetics, biotechnology and agri-food sectors. The international demand essentially involves nuts and butter, for which a new demand is emerging from small cosmetics companies in developed countries. It has a link to fair trade. Women's groups are the main stakeholders in shea butter processing. They are responding to this international demand, the volume of which remains limited to 10 percent of the local market. Competition among the groups for the international markets is fierce. Under what conditions can a "win-win" articulation be developed between the African industry stakeholders and those in developed countries? Women's groups seem to benefit from these exchanges. International trade in this product enables women's groups to gradually increase their bargaining power with international buyers while enhancing the logistics enabling them 


\section{Le beurre de karité, une production artisanale féminine}

\section{Le beurre de karité source de matière grasse en milieu rural}

L'arbre à karité ( Vitellaria paradoxa parkii ou butyrospermum parkii) pousse à l'état sauvage mais est entretenu par l'homme. La "ceinture du karité " comprend seize pays africains, les principaux producteurs étant le Nigeria (61\%), le Mali (12\%), le Burkina Faso (10\%), le Ghana (9\%). La production mondiale de karité est de 693000 tonnes en 2005 (CNUCED, 2009). L'aire de distribution du karité couvre $70 \%$ du territoire burkinabé. On peut le trouver sur les champs ou dans des zones forestières. La pulpe des fruits contient une noix, dont l'amande fournit le beurre, principale source de matière grasse pour les populations rurales dans les zones de production. Son arôme particulier est apprécié dans les plats traditionnels, mais pas forcément des jeunes générations urbanisées qui lui préfèrent l'huile végétale. Sa composition biochimique en fait une matière grasse de très bonne valeur nutritionnelle. Ses propriétés cosmétiques et pharmaceutiques sont nombreuses; il est aussi particulièrement populaire pour le massage des bébés. Le savon de karité évite notamment la déshydratation de la peau.

to guarantee the production quality and volume requirements in timeframes set by the customer. Still, there should be real pressure from consumers to ensure that the word "fair" is precisely defined, that it actually enables greater value for the grassroots labour input and that such can be duly confirmed. The limited volume opportunities and cutthroat competition among the groups must not be allowed to hamper local structuring of the commodity chain. These conclusions require a support mechanism for the women's groups so that the industry can be better controlled: processing, negotiation, products, quality, both for the export and local markets and those of the sub-region which are more accessible and less risky.

Key words: Burkina Faso; exports; market knowledge; shea butter; shea (tree).

Subjects: agrifood technologies; economy and rural development; processing, marketing; vegetal productions.

\section{Produire le beurre de karité : une activité artisanale féminine, à faible productivité}

Les filières du beurre de karité sont organisées par rapport aux circuits commerciaux. Le droit de collecte est lié à la propriété du champ si le karité pousse en zone cultivée, tandis que, dans les forêts spontanées, toutes les femmes ont le droit de collecter, selon la règle du "premier arrivé, premier servi".

Il existe ensuite plusieurs techniques pour conserver les noix. La qualité des amandes obtenues est fonction de la quantité de travail investie (USAID WATH, 2004) (tableau 1).

L'utilisation de l'une ou l'autre technique dépend de la destination des amandes vente ou transformation en beurre -, de la disponibilité de l'eau, du temps de travail, de l'accès à la formation aux techniques nouvelles, des opportunités commerciales. La transformation en beurre est longue et pénible (figure 1). Cinq femmes peuvent traiter $100 \mathrm{~kg}$ d'amandes en une journée de travail.

\section{De nouveaux acteurs dans la filière karité : les groupements de femmes}

L'essentiel du karité transformé en beurre au Burkina Faso passe par les femmes, qu'elles produisent pour elles-mêmes, pour commercialiser individuellement, ou au sein d'un groupement. Le groupement est à la fois une organisation pro- ductive, les femmes se regroupant pour satisfaire de grosses " commandes ", mais aussi un réseau de solidarité. Le fonctionnement de ces groupements a été étudié par de nombreux auteurs (Saussey, 2006 ; Elias et Carney, 2004). C'est à eux que la plupart des projets d'appui et la plus grosse partie de l'aide apportée à la filière karité sont destinés. Au Burkina, ces groupements sont regroupés en sept unions qui totalisent environ 250 groupements et regroupent de 30000 à 35000 femmes membres (tableau 2). Le Programme national karité (PNK), rattaché au Burkina à la présidence de la République, cherche à trouver une forme de représentation nationale, mais cette tâche n'est pas aisée.

\section{Transformation semi- industrielle au Burkina}

La transformation en beurre au Burkina concernait essentiellement deux unités industrielles dont l'activité de trituration du karité, moins rentable que celle d'autres oléagineux comme le coton, a décru depuis 10 ans, jusqu'à devenir aujourd'hui très faible ou nulle. Ces unités transformaient également le beurre de karité en savon de lessive ou de toilette, mais cette activité s'est aussi arrêtée ou ralentie. Dans l'espace économique laissé libre, des entreprises semi-industrielles trouvent un champ d'action à leur échelle. Le tableau 3 inventorie les sociétés de transformation.

La transformation industrielle du karité est en décroissance, alors que la transformation semi-industrielle par les groupements de femmes se maintient ou crôit. 
Tableau 1. Techniques de traitement des noix de karité.

Table 1. Shea almond production techniques.

\begin{tabular}{|c|c|c|c|}
\hline & $\begin{array}{l}\text { Noix bouillies (technique } \\
\text { améliorée fournissant } \\
\text { le meilleur beurre pour l'export) }\end{array}$ & $\begin{array}{c}\text { Noix fumées } \\
\text { (technique traditionnelle) }\end{array}$ & $\begin{array}{c}\text { Noix fermentées } \\
\text { (technique traditionnelle) }\end{array}$ \\
\hline Collecte des fruits & $\begin{array}{l}\text { Récolte des fruits mûrs tombés } \\
\text { de l'arbre }\end{array}$ & Récolte des fruits & Récolte des fruits \\
\hline Stockage & En fosses & En fosses & En fosses \\
\hline Dépulpage & $\begin{array}{l}\text { Après quelques jours de stockage } \\
\text { en fosse }\end{array}$ & $\begin{array}{l}\text { Après quelques jours de stockage } \\
\text { en fosse }\end{array}$ & \\
\hline $\begin{array}{l}\text { Chauffage/inactivation } \\
\text { des enzymes lipolytiques }\end{array}$ & Faire bouillir les noix dans l'eau & Fumer les noix dans un four à bois & \\
\hline Séchage & $\begin{array}{l}\text { Séchage des noix au soleil } \\
\text { Retourner régulièrement }\end{array}$ & & \\
\hline Décorticage & Manuel ou mécanique & & \\
\hline Séchage & $\begin{array}{l}\text { Séchage des noix au soleil ou four } \\
\text { amélioré (saison pluvieuse) }\end{array}$ & & \\
\hline \multirow[t]{2}{*}{ Stockage } & En sacs ou en greniers & En greniers & $\begin{array}{l}\text { Le fruit reste dans la fosse jusqu'à } \\
\text { commercialisation ou utilisation }\end{array}$ \\
\hline & Noix de karité prêtes à être comm & ercialisées ou transformées en beur & \\
\hline
\end{tabular}

Au total, la transformation du beurre de karité est une activité féminine pénible et peu rémunératrice, tant que les femmes n'ont pas accès à des technologies plus efficaces. Les groupements de femmes sont pourtant actifs dans ce domaine, car le karité est une des ressources naturelles les plus facilement accessibles pour les femmes pauvres en zone rurale, à qui il procure une activité, des revenus, et une insertion sociale au sein des groupements.

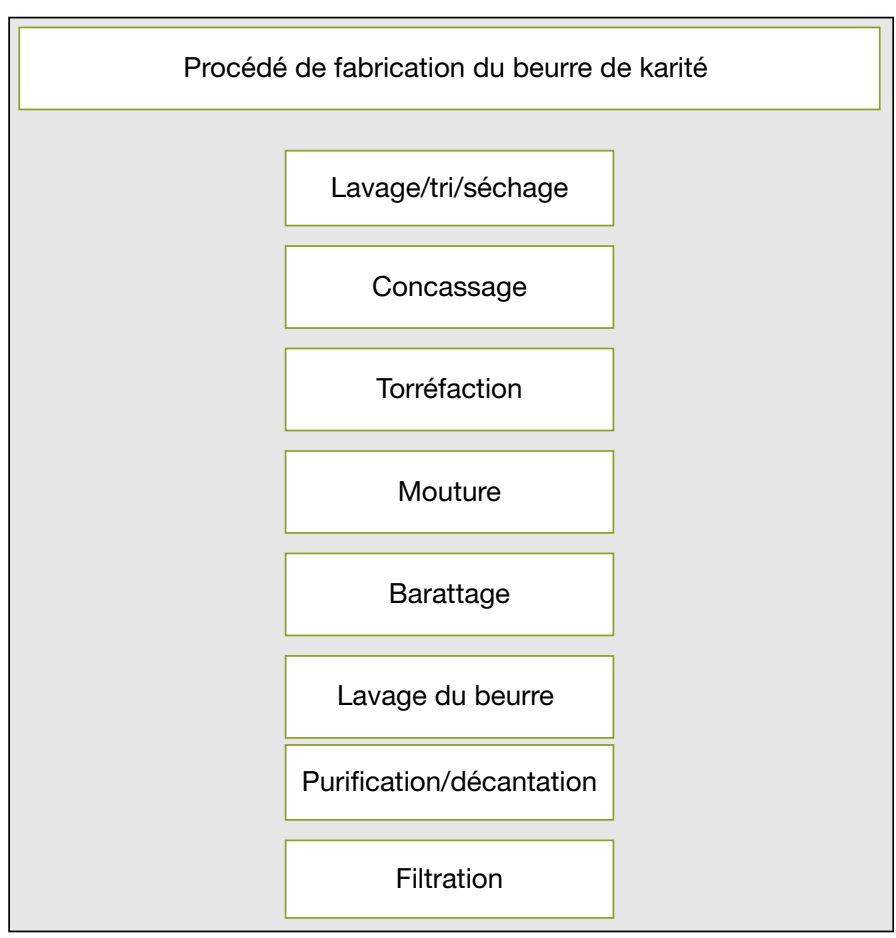

Figure 1. Procédé de transformation des amandes de karité en beurre.

\section{Entre sphère de l'échange et marché mondial : les filières du karité}

De façon schématique, les filières du karité peuvent être représentées comme décrit à la figure 2.

\section{Sphère locale de l'échange}

Sur les fruits récoltés, la plus grande partie, soit 80 à $90 \%$ selon les sources, est conservée au sein de la famille pour la transformation en beurre destiné à l'autoconsommation ou à la vente locale. Ces deux destinations du beurre représentent, pour les productrices, des débouchés sûrs et stables. Les profits sont minces mais certains. Le reste, soit $10 \%$ à $20 \%$ selon les sources, est commercialisé, soit sous forme de noix (pour la plus grande partie), soit sous forme de beurre. Les noix sont essentiellement destinées aux acheteurs internationaux. Certains acheteurs, opérant pour des compagnies internationales, achètent aussi des noix pour les transformer dans les pays voisins, comme le Ghana (Jones, 2006; Boffa et al., 1996).

La plus grande partie du beurre de karité commercialisé est destinée au marché local. Une enquête auprès de l'ensemble

Figure 1. Process for transforming shea almonds into butter. 
Tableau 2. Les Unions de groupements au Burkina Faso (Badini, 2003).

Table 2. Burkina Faso Group Unions (Badini, 2003).

\begin{tabular}{|c|c|c|c|}
\hline & Région & Nombre de membres & Siège \\
\hline $\begin{array}{l}\text { UFK : Union des femmes } \\
\text { productrices de karité }\end{array}$ & $\begin{array}{l}\text { Ouagadougou, Manga, Garango, } \\
\text { Tanghin Dassouri, Koudougou, Banfora }\end{array}$ & $\begin{array}{l}6 \text { unions de groupements de base, } \\
2000 \text { membres }\end{array}$ & Ouagadougou \\
\hline UGPPK Houet & Faramana, Koundougou & 16 groupements de base & Koundougou \\
\hline UGPPK Sissili / Siro & Sapouy, Léo & 12 groupements de base & Léo \\
\hline RENAPRO-K & & 11 groupements de base & Ouagadougou \\
\hline AFD Buyaba & $\begin{array}{l}\text { Tapoa, Komandjari, Kompienga, } \\
\text { Gnagna, Koulpeogo, Kadiogo }\end{array}$ & $\begin{array}{l}46 \text { groupements de base } \\
\text { soit } 5200 \text { membres }\end{array}$ & Fada Ngourma \\
\hline CDN & & 10 groupements de base & \\
\hline FADEFPSO & $\begin{array}{l}\text { Dano, Dissin, Diebougou, Gaoua, } \\
\text { Batié, Kampti }\end{array}$ & $\begin{array}{l}88 \text { groupements de base } \\
\text { soit } 20000 \text { membres }\end{array}$ & Dano \\
\hline
\end{tabular}

\section{Tableau 3. Unités de transformation industrielles et semi-industrielles au Burkina Faso.}

Table 3. Industrial and semi-industrial transformation units in Burkina Faso.

\begin{tabular}{|c|c|c|c|c|}
\hline Société & Échelle & Siège & Produits & Marché \\
\hline SOFIB & $\begin{array}{l}\text { Industrielle } \\
\text { Activité arrêtée pour le karité }\end{array}$ & Bobo Burkina & Savon de lessive & National \\
\hline SNCITEC & $\begin{array}{l}\text { Industrielle } \\
\text { Activité en ralentissement } \\
\text { concernant le karité }\end{array}$ & Bobo Burkina & $\begin{array}{l}\text { Beurre neutralisé } \\
\text { Savon de lessive }\end{array}$ & National et exportation \\
\hline Phycos & Semi-industrielle & Ouagadougou & $\begin{array}{l}\text { Savon de toilette, pommade, lait, } \\
\text { lotions, shampoings }\end{array}$ & National \\
\hline Karilor & Semi-industrielle & Ouagadougou & $\begin{array}{l}\text { Savon de toilette, beurre neutralisé } \\
\text { conditionné, pommades, shampoings, } \\
\text { lait, lotions }\end{array}$ & $\begin{array}{l}\text { National et exportation } \\
\text { Sous-région }\end{array}$ \\
\hline $\mathrm{SPH}$ & Semi-industrielle & Bobo Dioulasso & Savon de toilette, shampoing & $\begin{array}{l}\text { National et exportation } \\
\text { Sous-région }\end{array}$ \\
\hline Savomi & Semi-industrielle & Ouagadougou & Savon de toilette ou antiseptique & National \\
\hline Naturex & Semi-industrielle & Ouagadougou & Savon antiseptique & National \\
\hline
\end{tabular}

des marchés de la ville de Ouagadougou, réalisée sur une semaine, a permis d'en évaluer la taille : 10000 tonnes/an au Burkina Faso. Le beurre produit pour l'exportation représente environ 500 à 1000 tonnes, soit moins de $10 \%$ du beurre commercialisé sur les marchés locaux. Le marché local demeure le plus important débouché quantitatif pour les femmes, le plus accessible et le moins risqué, ce qui justifierait que plus d'intérêt lui soit accordé par les projets d'appui à la filière.

Les prix pratiqués dépendent de la saison, du conditionnement et de la localisation du marché. Ils varient entre 500 francs CFA/ $\mathrm{kg}$ (0,76 euro) et plus de 1200 francs CFA/kg (1,82 euro), selon les périodes et la qualité du beurre.

Le recensement des points de vente de karité à Ouagadougou compte 192 reven- deurs. La plupart de ces revendeurs sont situés sur les marchés (tableau 4).

L'enquête réalisée sur la ville de Ouagadougou donne la répartition suivante entre les différentes formes de commercialisation (tableau 5).

Les ventes sont donc essentiellement réalisées en grandes quantités, sous forme de yoruba, plat de beurre contenant entre 1,5 et $2,5 \mathrm{~kg}$, vendu entre 750 et 1750 francs CFA (1,14 euro et 2,66 euros) selon les vendeurs, la qualité et les périodes. Sur les marchés traditionnels, le beurre est aussi vendu en boules de 20 à 25 grammes au prix de 25 francs CFA (0,04 euro). La majeure partie des ventes passe par les circuits traditionnels, les étals sur les marchés. Les prix atteints par les produits atteignent presque ceux des commandes d'exportation. Les circuits qui permettent d'atteindre les couches aisées de la population correspondant aux " nouveaux marchés urbains "sont très peu représentés. Les leaders des groupements de femmes et les grands commerçants en beurre de karité ne semblent pas s'y intéresser.

\section{Des filières d'exportation très concentrées}

Le commerce international du karité concerne essentiellement les noix et aujourd'hui accessoirement le beurre. Il porte sur environ $10 \%$ des noix disponibles, avec de fortes variations selon les années. Deux intervenants majeurs au Burkina : Aarhus et Loders Croklaan, les plus grands fabricants de CBE (Cocoa Butter Equivalent) en Europe dominent ce commerce particulièrement opaque en 


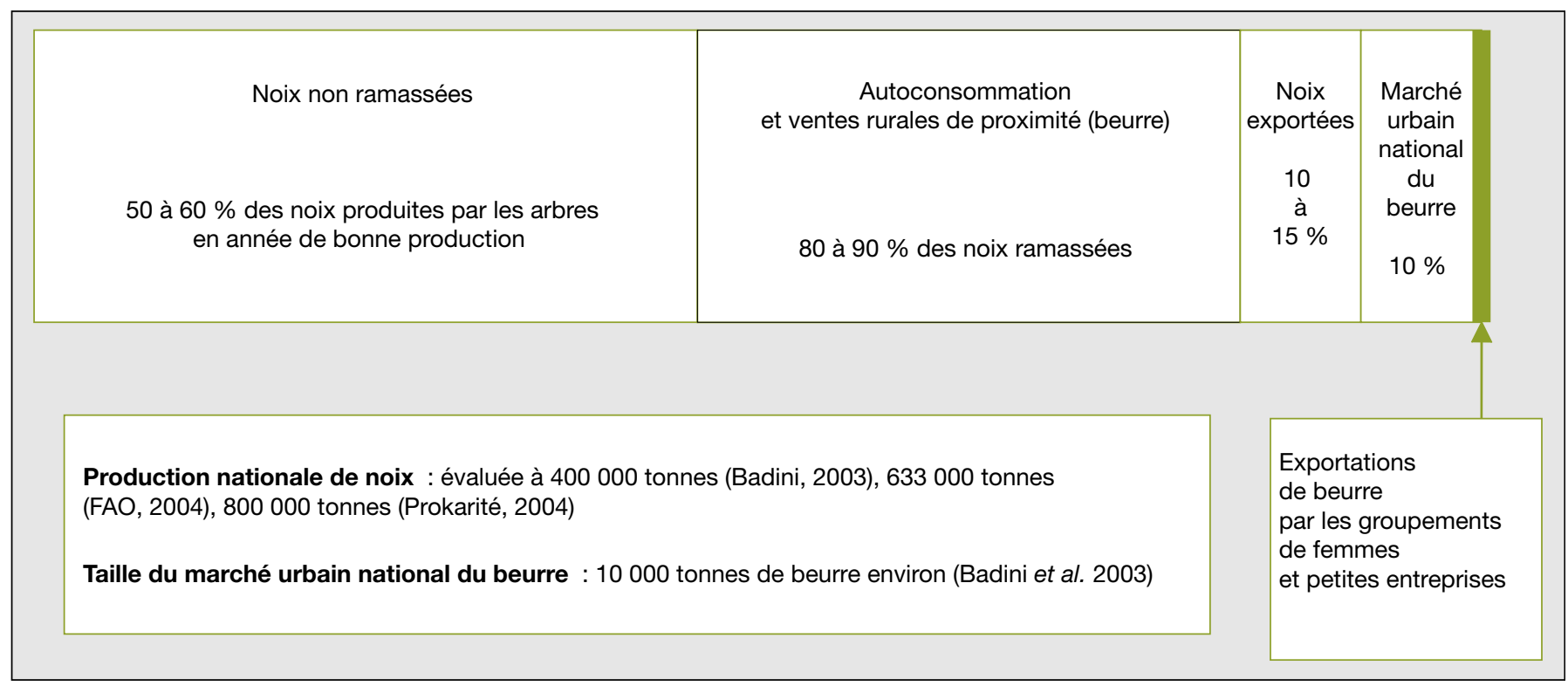

Figure 2. Importance relative des différents marchés des noix de karité au Burkina Faso

Figure 2. Comparative importance of the different shea nut markets for Burkina Faso.

\section{Tableau 4. Les circuits de vente du beurre de karité sur le marché de Ouagadougou.}

Table 4. Shea butter sales circuit on the Ouagodougou market.

\begin{tabular}{lc}
\hline Nature du commerce & Nombre de points de vente \\
\hline Étal sur les marchés traditionnels & 118 \\
Détaillant : hangar ou kiosque & 39 \\
Boutique de quartier & 15 \\
Supermarché/supérette & 6 \\
Magasin spécialisé & 0 \\
Vendeur ambulant & 13 \\
Autres & 1 \\
\hline
\end{tabular}

\section{Tableau 5. Volumes, prix et conditionnements du beurre de karité vendu sur les marchés à Ouagadougou.}

Table 5. Volumes, prices and conditionings of shea butter sold at Ouagodougou markets.

\begin{tabular}{lcc}
\hline Type de conditionnement & $\begin{array}{c}\text { Volume écoulé } \\
\text { en une semaine }(\mathbf{k g})\end{array}$ & Prix par kg \\
\hline Beurre en boule de 25 grammes & 290 & 1000 francs CFA $/ \mathrm{kg}$ \\
Beurre en yoruba & 1403 & 500 à 700 francs CFA/kg \\
Beurre conditionné en pots & 212 & 1000 francs CFA $/ \mathrm{kg}$ \\
Beurre conditionné en sachets & 7 & 1000 francs CFA $/ \mathrm{kg}$ \\
\hline
\end{tabular}

achetant les noix à des commerçants locaux au travers de sociétés internationales fournisseuses de produits agricoles, ou par leur propre réseau de collecteurs.
Les quantités échangées ne sont pas connues et les données existantes ne sont pas fiables (Collinson et Zewdie Bosuener, 1999).

\section{Un marché naissant d'exportation directe par les groupements de femmes}

Les groupements de femmes jouent un rôle croissant dans l'exportation du beurre. Ces dernières années, plusieurs petites entreprises cosmétiques européennes, japonaises ou des États-Unis, voire même des firmes internationales, ont commencé à acheter le beurre directement aux groupements de femmes en se réclamant du commerce équitable. Chaque année, les quantités commercialisées en Europe par ce créneau représentent au maximum 500 tonnes de beurre pour le Burkina Faso. Ces commandes sont importantes pour les groupements, car elles représentent près de la moitié de leurs exportations. Les prix pratiqués dépendent de chaque client et des caractéristiques spécifiques du beurre acheté. Les prix varient de moins de 1000 francs CFA/ kg (1,52 euro) à plus de 1200 francs $\mathrm{CFA} / \mathrm{kg}$ (1,82 euro), voire même dans certains cas 2500 francs CFA/ kg (3,81 euros) pour du beurre "biologique " conditionné au départ de Ouagadougou.

\footnotetext{
${ }^{1}$ Le karité est un produit de cueillette, mais certains karités poussent sur des champs de coton qui reçoivent des intrants chimiques. De ce fait, tout le beurre de karité produit au Burkina n'est pas forcément "biologique ".
} 
Le volume des commandes varie de quelques tonnes à 200 tonnes au maximum. Ces achats directs de beurre aux groupements de femmes ont un effet positif, car ils permettent aux groupements de s'organiser au niveau logistique, et les rendent capables, par l'expérience, de fournir des quantités conséquentes et d'améliorer la qualité du beurre. Cette expérience acquise au travers de la collaboration avec de petites entreprises étrangères devrait ensuite leur être utile pour mieux se positionner sur les marchés urbains nationaux ou l'exportation dans la sous-région.

Cependant, dans la mesure où ces commandes ont encore un volume très limité par rapport à leur capacité de production, les leaders des groupements luttent pour les capter. Une commande représente un revenu important qui passe au travers du groupement et une opportunité d'emplois pour de nombreuses femmes.

\section{Braudel et le karité : les trois étages de l'économie}

L'analyse que fait Braudel de la dynamique du capitalisme et la distinction qu'il opère entre échanges de proximité et échanges lointains s'adaptent parfaitement à la filière karité (Braudel, 1985). Les échanges commerciaux concernant le karité se répartissent en deux catégories.

Dans la première, on repère les échanges quotidiens du marché, les ventes locales ou à faible distance. Des échanges sans surprise, "transparents", dont chacun connaît à l'avance les tenants et les aboutissants et dont on peut supputer à peu près les bénéfices, toujours mesurés. La marge bénéficiaire est à la fois sûre et modérée. On trouve ici les ventes de beurre de karité en boules ou yorubas, le savon. Les prix sont relativement modérés (300 à 500 francs $\mathrm{CFA} / \mathrm{kg}, 0,45$ à 0,76 euro $/ \mathrm{kg}$ ), mais ces prix couvrent les coûts de production et permettent une marge si les noix sont récoltées par les femmes transformatrices, ou si elles sont achetées au moment des cours favorables. Les quantités concernées sont importantes; les opportunités commerciales sont proches et relativement dénués de risques.

Mais à côté du marché du karité se dessine ce que Braudel appelle le contre-marché. Ne cherche-t-il pas à se débarrasser des règles du marché traditionnel, paralysantes à l'excès? Des marchands itinérants, ramasseurs, collecteurs de noix rejoignent les producteurs chez eux. Ils achètent directement les noix, éventuellement après d'âpres négociations tenant compte du dénuement particulier des femmes au moment de la "soudure ". Ce type d'échange substitue aux conditions normales du marché collectif - où l'information est relativement partagée par les acteurs susceptibles de mener les transactions (producteurs, commerçants) - des transactions individuelles dont les termes varient arbitrairement selon la position respective des intéressés. Les échanges les plus inéquitables sont alors possibles : Dans le domaine du karité, ceci signifie que les collecteurs achètent aux plus bas prix aux femmes isolées en zone rurale. Il est évident qu'il s'agit d'échanges inégaux où la concurrence, loi essentielle de l'économie de marché, l'équilibre entre offre et demande à l'origine de la fixation des prix ne tiennent plus de place.

L'acheteur dispose de deux avantages :

- il a rompu la relation entre le producteur et celui à qui est destinée la marchandise: il est seul à connaître les conditions du marché aux deux bouts de la chaîne ;

- il dispose d'argent comptant. La contrainte financière qui pèse sur les femmes qui ont des besoins financiers à court terme les met en position de "price takers " par rapport aux acheteurs de noix. Ce système de commerce international portant sur les matières premières forme de longues chaînes marchandes que les historiens appellent le "fernhandel" un domaine de libre manœuvre, qui opère sur des distances qui le mettent à l'abri des surveillances ordinaires ou lui permettent de les contourner. N'entre pas qui veut dans cette activité. Ces acteurs ont la supériorité de l'information, de la culture et ils sont seuls à avoir l'intelligence du marché mondial et de la valeur du produit sur ce marché. Qu'ils aient des monopoles ou simplement la puissance nécessaire pour effacer la concurrence, qui en douterait? (Vershave, 2005).

\section{L'espace mondial peut-il être positif pour l'économie domestique du karité ?}

Souvent, lorsqu'une activité devient rentable, les hommes s'en emparent au détriment des femmes. Au contraire, la pénibilité des tâches de ramassage et de fabrication du beurre fait que la filière karité est encore tenue par des femmes
(Saussey, 2006). La littérature accorde une place très importante aux exportations de beurre par les groupements. Le danger, c'est évidemment de ne voir que cette filière, de la décrire avec un luxe de détail qui suggère une présence envahissante, alors qu'elle n'est qu'un fragment d'un vaste ensemble.

Plus que de contribuer à organiser la filière du beurre de karité au niveau local, cette opportunité - qui porte sur des volumes faibles par rapport aux capacités de production des groupements - contribue à créer une concurrence exacerbée entre les principales actrices que sont les leaders des groupements de femmes. Il s'agit pour chacune de gagner une ou plusieurs commandes " équitables ", afin d'améliorer la situation financière du groupement. Cette concurrence féroce ne facilite pas la naissance d'organisations professionnelles, pourtant nécessaires à la filière : négociations sur les normes, emballages, raffinage du beurre, purification....

Pour que ces commandes directes puissent maintenant jouer un rôle positif, dans le sens du renforcement du rôle des femmes dans la filière, le marché du beurre équitable, ou l'achat direct aux femmes, doit " sortir de la niche ", concerner des quantités conséquentes, et envisager des liens avec le marché local.

\section{Conclusion}

Le beurre de karité est un exemple remarquable d'une articulation de fait entre trois sphères de circulation : celle de l'autoconsommation, celle du marché local, proche et urbain, et celle du marché international.

Certainement des voies sont possibles vers une meilleure valeur ajoutée pour les groupements si :

- Il existe une pression effective des consommateurs pour que le mot "équitable "soit précisément défini, qu'il permette effectivement une meilleure valorisation du travail à la base, et qu'existe la capacité de le vérifier. Ceci pose la question du contenu d'une norme sur le commerce équitable ;

- les groupements de femmes acquièrent progressivement de meilleures capacités de négociation avec les acheteurs internationaux, en même temps que la capacité logistique de fournir la qualité et les volumes requis dans les délais fixés par le client. Les commandes de beurre passées 
dans le cadre du commerce équitable contribuent à améliorer l'organisation et la compétence des femmes dans les groupements, alors que les femmes sont en position de price takers dans l'achat de noix pour le marché international.

Toutefois, il reste que cette capacité d'exportation directe du beurre de karité ne doit pas masquer les opportunités du marché national, où les potentiels de développement sont plus grands et plus accessibles.

Ces conclusions appellent à un processus d'appui aux groupements de femmes pour une meilleure maitrise de cette filière : transformation, négociation, produits, qualité, plutôt que des appuis aux projets et des ONG ciblés sur un ou plusieurs groupements en particulier. Un appui plus global pourrait améliorer la capacité des groupements à mieux gérer leur insertion internationale. La question des normes et des prix peut, à l'inverse, aider à structurer le marché national. Ces développements, sur le marché national comme sur le marché international, ne sont possibles que si la productivité est accrue dans la filière et que la qualité est soutenue par les groupements. Il s'agit là d'un enjeu technologique sur lequel le Groupe de recherche et d'échanges technologiques (Gret) travaille également en mettant au point des techniques de transformation et d'analyse innovantes.

\section{Remerciements}

Ces résultats sont issus d'un programme de recherche de l'Union européenne coordonné par le Groupe de recherche et d'échanges technologiques (Gret) avec les partenaires Sicarex (Burkina Faso), 2IE (Burkina Faso), IC Sahel (Mali), Inde (Portugal) et Agir (France).

\section{Références}

Boffa JM, et al. Shea nut production and collection in agroforestry parklands of Burkina Faso. Rome: FAO, 1996.
Braudel F. La dynamique du capitalisme. Paris: Flammarion, 1985.

CNUCED L'information de marché dans le secteur des produits de base. Genève: CNUCED, 2009. http://www.unctad.org/infocomm/francais/karite/prix.htm

Collinson C. Zewdie-BosuenerA. Shea butter markets : Their implication for Ghanaian shea butter exporters. Washington: CGIAR, 1999.

Elias M, Carney J. La filière feminine du karite : productrices burkinabè, "éco consommatrices" occidentales et commerce équitable. Cahiers de géographie du Québec 2004; 48 : 71-88.

Jones A. Market research on the shea nuts and shea butter in the international market. Utrecht: ICCO, 2006.

Saussey M. Les associations féminines de productrices de beurre de karité au Burkina Faso: Enjeux de développement durable. In York University ed. Annual meeting of the Canadian Association for studies in co-opération, Congress or the social sciences and humanities. Toronto : York University, 2006.

USAID. The shea butter value chain: Production, transformation and marketing in West Africa. Accra : WATH, 2004.

Vershave FX. La maison monde. Paris: ECLM, 2005. 